\title{
Adaptive Optimization for OpenCL Programs on Embedded Heterogeneous Systems
}

\author{
Ben Taylor, Vicent Sanz Marco, Zheng Wang * \\ School of Computing and Communications, Lancaster University, UK \\ \{b.d.taylor, v.sanzmarco, z.wang\}@lancaster.ac.uk
}

\begin{abstract}
Heterogeneous multi-core architectures consisting of CPUs and GPUs are commonplace in today's embedded systems. These architectures offer potential for energy efficient computing if the application task is mapped to the right core. Realizing such potential is challenging due to the complex and evolving nature of hardware and applications. This paper presents an automatic approach to map OPENCL kernels onto heterogeneous multi-cores for a given optimization criterion - whether it is faster runtime, lower energy consumption or a trade-off between them. This is achieved by developing a machine learning based approach to predict which processor to use to run the OPENCL kernel and the host program, and at what frequency the processor should operate. Instead of hand-tuning a model for each optimization metric, we use machine learning to develop a unified framework that first automatically learns the optimization heuristic for each metric off-line, then uses the learned knowledge to schedule OPENCL kernels at runtime based on code and runtime information of the program. We apply our approach to a set of representative OPENCL benchmarks and evaluate it on an ARM big.LITTLE mobile platform. Our approach achieves over $93 \%$ of the performance delivered by a perfect predictor. We obtain, on average, 1.2x, 1.6x, and 1.8x improvement respectively for runtime, energy consumption and the energy delay product when compared to a comparative heterogeneous-aware OPENCL task mapping scheme.
\end{abstract}

Keywords Heterogeneous Multi-cores, Predictive Modeling, Energy Efficiency, OpenCL

\section{Introduction}

Embedded systems with heterogeneous multi-cores are commonplace. These systems offer the potential of energy efficient computing via diverse processing units specially tuned for a certain class of workloads and for power or performance. While promising, to unlock the potential of such systems, software must adapt to the variety of processors, knowing what type of processors to use and at what frequency the processor should operate.

\footnotetext{
${ }^{*}$ Corresponding author: Zheng Wang (z.wang@lancaster.ac.uk).
}

OPENCL has emerged as a standard programming model for heterogeneous systems. It allows the same code to be executed across a variety of processors including CPUs, GPUs and DSPs. While OPENCL provides functional portability, its performance often varies across different processing units [13, 19, 26]. Therefore, there is a need to determine where and how to map a OPENCL task to best utilize the underlying heterogeneous hardware resources.

In this paper, we tackle the issue of OPENCL task mapping across heterogeneous CPUs and GPUs for multiple optimization criteria. Although task scheduling is a heavily studied area $[3,11$, $34,35]$, heterogeneous task mapping is made more complex by the different runtime and energy characteristics an OPENCL kernel will experience on different processing units $[9,28,30]$. While manually task mapping may be possible in certain domains [2, 4], it is not the general case as the decision will depend on: the application itself, the program input, underlying hardware, and optimization criteria. Given that embedded systems are becoming increasingly diverse with ever-changing hardware and software, finding the right mapping for a single application may have to be repeated many times throughout its lifetime, hence, making automatic approaches attractive.

This paper presents a novel compiler-based approach to map and schedule OPENCL tasks across embedded heterogeneous computing units. We do so by employing machine learning techniques to automatically construct predictors to determine at runtime the optimal processor configuration - which processing units and clock frequencies to use to run the given OPENCL driver program and kernel function, taking into consideration the optimization criterion - whether it is faster response time, lower energy consumption or a trade-off between them. Our predictors are first trained off-line. Then, using code and data features extracted from the compiler intermediate representations (IR), the models predict the optimal processor configuration for a new, unseen program, depending on what we want to optimize for. We show that our unified machine learning based framework can automatically derive high-quality optimization heuristics across different optimization metrics, despite the optimal configurations changing from one metric to the other. Our approach avoids the pitfalls of using a hard-wired heuristics that require human modification every time the optimization criterion or hardware changes.

We apply our approach to 15 OPENCL benchmarks and evaluate them on a representative big.LITTLE mobile platform, using three metrics: runtime, energy consumption and the energy delay product. We compare it to a comparative machine learning based mapping scheme [13]. We show that our approach delivers, on average, over $1.2 \mathrm{x}$ improvement across all benchmarks.

This paper makes the following two main contributions:

- We develop a unified machine learning based framework for mapping OPENCL tasks on embedded heterogeneous multi-core platforms across optimization criteria; 
- We show that our automatic approach constantly outperforms comparative work across all evaluation metrics.

\section{Related Work}

Our work lies at the intersection of numerous areas: GPU performance optimization, GPU task scheduling, predictive modeling and energy optimization.

GPU Performance Optimization There is an extensive body of work looking at GPU performance optimization through the use of compilation and runtime techniques [22, 31, 38]. Most prior work in the field uses analytical models to determine optimization strategies. contrast, our work employs machine learning to automatically construct models for different optimization goals. Prior studies show that portable mapping techniques are important, program performance depends on the underlying hardware $[19,26]$. Our work is among attempts to create portable mapping techniques.

GPU Task Scheduling Numerous approaches have been proposed to map OPENCL or CUDA kernels on heterogeneous computing devices. Some of the prior work uses profile runs to partition work across the CPU and the GPU [1, 13, 20, 23]. In contrast to these works, our approach does not rely on profiling information, therefore, has less runtime overhead. Other work considers code and data transformations for CPU/GPU architectures [21, 27], GPU task preemption [6], and multi-task scheduling [10, 14-16, 22, 43]. These prior works primarily target performance optimization, while this work aims to build a portable approach for arbitrary optimization goals. Moreover, existing approaches on multi-task scheduling are orthogonal to our work.

Predictive Modeling Machine learning based predictive modeling is emerging as a powerful technique for optimizing parallel programs [28, 29, 33, 37, 39-41]. Its great advantage is its ability to adapt to changing platforms as it has no a prior assumptions about their behavior. The work presented by Grewe et al. is the nearest work [13, 42], which builds a decision tree model to predict where (CPU or GPU) to run a given OPENCL kernel. Our approach differs from this work in two aspects. Firstly, our approach can adapt to different optimization goals while [13] only targets runtime. Secondly, our approach considers the clock frequency of the processors while [13] does not.

Energy Optimization How to effectively exploit heterogeneous architectures for energy efficient computing is a heavily studied area. Some of the recent examples in the area include: how to distribute workloads across CPUs and GPUs [25] and MPSoCs [4], power modeling for GPGPUs [24], and energy aware iterative compilation $[7,12]$ etc. Unlike these approaches which all use analytic models or hard-wired heuristics to perform optimization for a specific goal, we develop a portable method that can automatically re-target for any optimization metric. Our approach shares the same spirit as the work presented by Ren et al. [32], we both use machine learning to build predictive models for energy optimization. However, we target optimizing OPENCL programs on heterogeneous systems, while [32] focuses on scheduling mobile web browsing processes on CPUs.

\section{Background}

\subsection{Problem Definition}

Scope This work aims to develop a runtime system that can adapt to arbitrary optimization goals for OPENCL kernel mapping. An OPENCL program typically consists of two parts, a kernel program (which might contain several OPENCL kernel functions) to run on an OPENCL-compatible device (in our case a CPU or GPU), and a driver (or host) program to run on the general purpose $\mathrm{CPU}$ to offload the kernel computation. OPENCL kernel functions are compiled at runtime on the host $\mathrm{CPU}$ and sent to execute on the compatible device. This work is concerned with determining the best processor configuration - that is - which processor to run the driver program and the kernel, and at what clock frequency the processing units should operate.

Notation Our configuration notation is HostDev $\cdot b / L$, freq $_{\text {- }}$ KernelDev $\cdot b / L$, where $b$ and $L$ stands for big and little CPU/GPU respectively. For example, notation, $C P U_{L, 800 M h z}-G P U_{b}$, means running the host program on the little CPU at $800 \mathrm{Mhz}$ and the kernel on the big GPU. It is to note that we found that using the default frequency to run the OPENCL kernel gives the best performance, so we do not configure the clock frequency for running the kernel.

Optimization Metrics Unlike prior work [13], we do not just develop a method to optimize runtime. Instead, we want to develop a methodology that can be used for any optimization goal. For the purpose of evaluation, we target three lower is better metrics: (a) runtime, which aims to execute the kernel function as fast as possible; (b) energy consumption, which aims to use as little energy as possible; and (c) energy delay product (EDP), calculated as energy $\times$ runtime, which aims to find a balance between both energy consumption and performance.

Hardware platform Our work targets the ARM big.LITTLE architecture. These platforms couple a energy-tuned processor (LITTLE) with a faster and more power-hungry processor (big), some also come with a GPU which can be used for general purpose computing. The uniqueness of each processing unit allows for much more energy efficient computing when utilized to its full potential. Our work is evaluated on an Odroid-Xu3 embedded development board. We chose this platform as it is: representative of ARM big.LITTLE mobile architectures, provides us with an OPENCL implementation for both the CPU and GPU, and allows us to extract real-time energy readings using the on-board energy sensors. The architecture used in the Odroid system is used in multiple mobile devices such as the Samsung Galaxy S5.

\subsection{Motivating Example}

Consider scheduling four OPENCL kernels from the Rodinia benchmark suite on an ARM big.LITTLE platform with heterogeneous processing units. These kernels can be run on either of the heterogeneous CPU clusters, a Cortex-A15 processor (big) and a Cortex-A7 processor (little), or the Mali-T628 GPU. The OPENCL libraries recognise the Mali-T628 as two separate GPUs, one containing 4 cores (big), the other containing two (little).

Table 1 lists the best processor configurations for each of the three metrics considered in this work. To obtain the optimum processor configurations we performed an exhaustive search across all configurations, and monitored the energy consumption and runtime of each. We then calculated the processor configuration which produced the minimum value (as each metric is lower-isbetter) for each metric and kernel combination.

Figure 1 compares the potential improvement of each kernel and metric over a baseline that runs all kernels on the big GPU, $C P U_{B, 2.0 G h z}-G P U_{b}$. For runtime, the baseline performs well for BFS_1 and Nearest Neighbor, but a speed-up of $51 \%$ and $80 \%$ can be achieved for find_index and lud_diagonal respectively when the optimum configuration is used. Using the big GPU leads to significant energy consumption. For this metric, the best configuration gives over $49 \%$ (up to $95 \%$ ) reduction for each of the four benchmarks. Finally, for EDP, the best configuration gives over $46 \%$ (up to $98 \%$ ) reduction for those kernels. Clearly, there is significant room for improvement over simply running the kernel on the GPU, and the best processor configuration could change from one optimizing metric to another. 
Table 1: The best configuration which optimizes for each of the metrics: Energy Consumption, Runtime, and EDP.

\begin{tabular}{llll}
\hline Kernel & Energy Consumption & Runtime & EDP \\
\hline BFS_1 & $C P U_{L, 800 M h z}-G P U_{b}$ & $C P U_{L, 1.4 G h z}-G P U_{b}$ & $C P U_{L, 1.2 G h z}-G P U_{b}$ \\
find_index & $C P U_{L, 800 M h z}-C P U_{l}$ & $C P U_{B, 2.0 G h z}-C P U_{b}$ & $C P U_{B, 1.6 G h z}-C P U_{b}$ \\
Nearest Neighbor & $C P U_{L, 1.0 G h z}-G P U_{l}$ & $C P U_{B, 2.0 G h z}-G P U_{b}$ & $C P U_{L, 1.4 G h z}-G P U_{l}$ \\
lud_diagonal & $C P U_{L, 800 M h z}-C P U_{l}$ & $C P U_{B, 2.0 G h z}-C P U_{b}$ & $C P U_{L, 1.2 G h z}-C P U_{l}$ \\
\hline
\end{tabular}

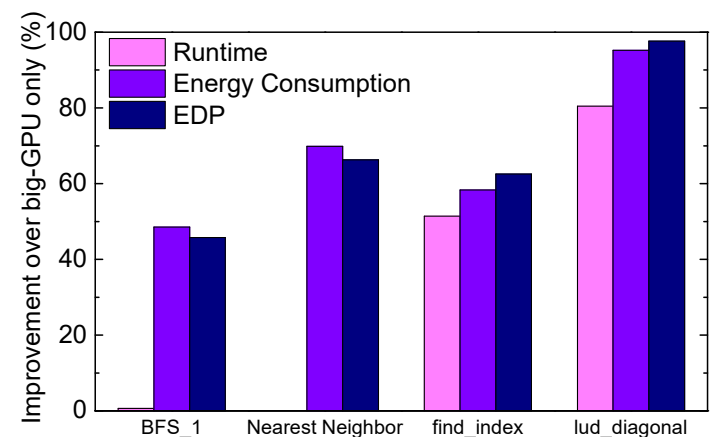

Figure 1: The percentage decrease (higher is better) of different metrics achieved when choosing the optimum configuration. The baseline is offloading each kernel to the big GPU, $C P U_{B, 2.0 G h z}-$ $G P U_{b}$.

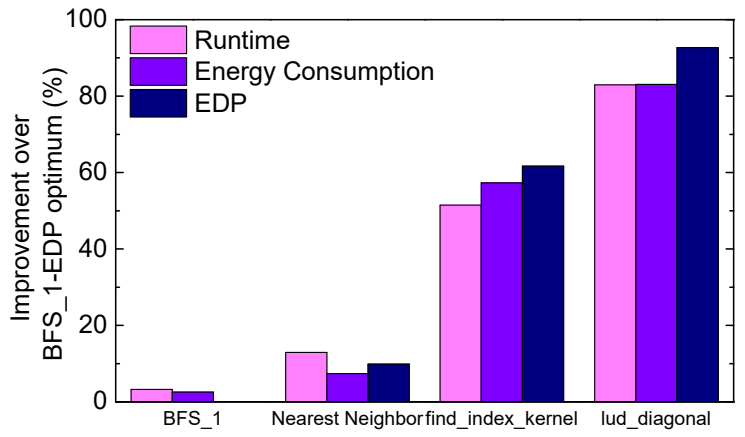

Figure 2: Available room for improvement when using the optimum configuration found for BFS_1 when optimizing for EDP, $C P U_{L, 1.2 G h z}-G P U_{b}$. This diagram shows that the best configuration varies across metrics and programs.

Figure 2 normalizes the best available performance of each metric for find_index, lud_diagonal, and Nearest Neighbor to the performance achieved by using the best configuration found for BFS_1, when optimizing for EDP (BFS_1-EDP). It shows that the best configuration also changes from one kernel to another; in other words no one configuration can be used to optimize for all kernels and all metrics. Overall, the best configuration for BFS_1-EDP provides a near optimal choice across all metrics for BFS_1. Nearest Neighbor also achieves near optimum results across all metrics for this configuration; although, a improvement of 13\%, 7\%, and $9 \%$ can still be achieved for runtime, energy consumption and EDP respectively. Kernels find_index and lud_diagonal achieve far from optimal results under this configuration. The best configuration is able to give an improvement between $51 \%$ and $92 \%$ (average of $71 \%$ ) for all metrics. Therefore, finding an optimal configuration for one kernel and applying it to all others is likely to miss significant optimization opportunities. This example demonstrates that choosing the right processor setting has a significant impact on the resultant performance and energy consumption, and the optimal configuration must be determined on a per-kernel and per-optimization-goal basis

Attempting to find the optimum configuration through means of an exhaustive search would be ineffective, the overhead involved would be far bigger then the potential benefits. Classical hand-

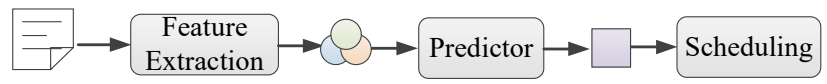

kernels feature values processor config.

Figure 3: Overview of our approach.

written heuristics are not ideal either, as they are not only complex to develop, but are likely to fail due to the variety of programs and evolving OPENCL devices. An alternate approach, and the one we chose to use, is to use machine learning to automatically construct predictive models for determining processor configurations, providing minimal runtime and development overhead.

\section{Overall Methodology}

Our approach takes a new, unseen OPENCL kernel function and is able to predict the optimum, or near optimum, processor configuration. An overview of our approach can be seen in Figure 3, and is described in more detail in Section 5.1.

When an OPENCL kernel is launched, our approach will collect a set of information or features to capture the characteristics of the kernel. As OPENCL is just-in-time compiled, the set of feature values collected by our approach can reflect the program input that is available at runtime. Feature extraction is performed on the LLVM intermediate represenations (IR); we only use code features and do not profile the program. Table 2 presents a full list of all our considered features. After collecting the feature values, a machine learning based predictor (that is trained offline) takes in the feature values and predicts which processor configuration should be used to execute the OPENCL kernel. This prediction includes the choice of host processor and its configuration in conjunction with the choice of accelerator. Finally, when the OPENCL kernel is ready to be offloaded to an accelerator we configure the host processor and offload the kernel to the predicted processor.

\section{Predictive Modelling}

Our model for predicting the best processor configuration is comprised of a set of Support Vector Machines (SVMs) arranged a hierarchically, show in Figure 4. Varying degrees of the polynomial kernel is used for each of our SVMs. We have evaluated a number of alternate modelling techniques, including regression, Naive Bayes, K-Nearest neighbour, decision trees, and artificial neural networks (see also Section 8.2). We chose SVM because it gives the best performance and can model both linear and non-linear problems. The input to our model is a set of features extracted from the target OPENCL kernel function. The output of our model is a label that indicates the optimal processor configuration to the host program and the OPENCL kernel, including the frequency of the host processing unit.

Building and using such a model follows the 3-step process for supervised machine learning: (i) generate training data (ii) train a predictive model (iii) use the predictor, described as follows.

\subsection{Model Description}

Figure 4 gives an overview of our predictive model. We chose a hierarchical layout as it yields the best results for our training data. In total we use 4 SVMs in our predictive model, and each has been given a name to allow for clear references. Below we will give a brief description of each SVM in our model. 
Table 2: Raw features we considered using in this work.

\begin{tabular}{|c|c|c|}
\hline Property & Feature & Description \\
\hline program structure & $\begin{array}{l}\text { avg_BB_preds } \\
\text { avg_BB_succ } \\
\text { num_edges } \\
\text { num_BB } \\
\text { avg_phi_args } \\
\text { BB_with_0_phi } \\
\text { num_instr } \\
\text { num_cond_br } \\
\text { num_uncond_br } \\
\text { num_br }\end{array}$ & $\begin{array}{l}\text { Average \# of predecessors to a basic block } \\
\text { Average \# of successors to a basic blocks } \\
\text { \# of edges in the Control Flow Graph (CFG) } \\
\text { \# of basic blocks } \\
\text { Average \# arguments to a phi nodes } \\
\text { \# basic blocks with } 0 \text { phi nodes } \\
\text { \# instructions } \\
\text { \# conditional branch instructions } \\
\text { \# unconditional branch instructions } \\
\text { \# branch instructions }\end{array}$ \\
\hline types of computation & $\begin{array}{l}\text { num_int_bin_ops } \\
\text { num_bin_ops } \\
\text { num_unary_instr }\end{array}$ & $\begin{array}{l}\text { \# binary operations with ints } \\
\text { \# binary operations } \\
\text { \# unary instructions }\end{array}$ \\
\hline amount of computation & num_comp & \# compute operations \\
\hline types of memory access & $\begin{array}{l}\text { num_mem } \\
\text { num_localmem }\end{array}$ & $\begin{array}{l}\text { \# accesses to global memory } \\
\text { \# accesses to local memory }\end{array}$ \\
\hline amount of memory accesses & $\begin{array}{l}\text { num_load } \\
\text { num_store }\end{array}$ & $\begin{array}{l}\text { \# load instructions } \\
\text { \# store instructions }\end{array}$ \\
\hline layout of memory accesses & num_coalesced & \# coalesced memory accesses \\
\hline data layout & $\begin{array}{l}\text { avg_work_size } \\
\text { transfer_size }\end{array}$ & $\begin{array}{l}\text { Average work size of kernels in a benchmark } \\
\text { \# bytes transferred to the accelerator }\end{array}$ \\
\hline data types used & num_float_inst & \# instructions which use floats \\
\hline
\end{tabular}

Table 3: Combined features used in this work.

\begin{tabular}{lll}
\hline Property & Feature & Calculated \\
\hline Ratio of memory access per work size & mem_by_work & (num_localmem + num_mem) *avg_work_size \\
Ratio of computation to memory transfer & comm-comp_ratio & transfer_size / $($ num_mem + num_comp) \\
Ratio of memory access to computation & comp-mem_ratio & num_comp/num_mem \\
Percentage of coalesced memory access & perc_coal_mem_acc & num_coalesced/num_mem \\
Percentage or memory accesses per instruction & mem_acc_per_instr & (num_load + num_store) /num_instr \\
Ratio of transfer size to work size & transfer-work_ratio & transfer_size/avg_work_size \\
\hline
\end{tabular}

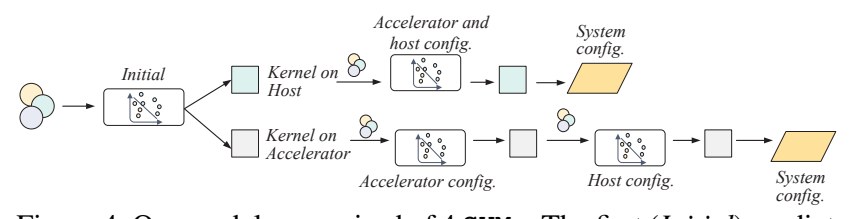

Figure 4: Our model, comprised of 4 SVMs. The first (Initial) predicts which type of device to run a kernel. The result then defines which branch we take through our model. Each branch predicts the system configuration for the given kernel. See Section 5.1.

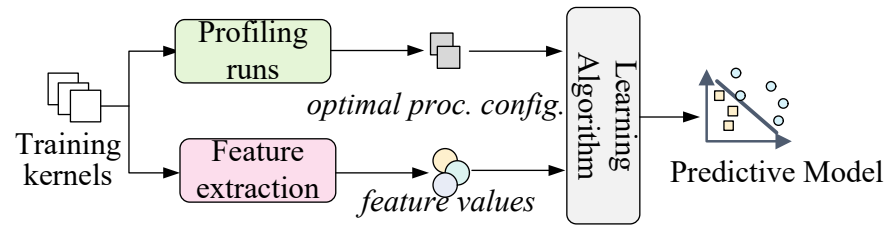

Figure 5: The training process. Our unified machine learning framework uses the same procedure to train a hierarchical predictive model for each optimization goal.

Initial The first SVM in our model, all data which enters our model passes this SVM first. Initial is used to place each kernel into one of two groups: accelerate-on-host and accelerate-off-host, which will be passed to the Accelerator and Host Config and Accelerator Config SVMs respectively. Each group requires a different approach to predict the optimum processor configuration for a kernel, hence our use of multiple branches.

Accelerator and Host Config The OPENCL API for our system recognises both CPUs as one processor, this resulted in us being unable to separate the host and kernel processes. As a result, when a kernel runs on the host it must adopt the same processor set-up as its host process. Accelerator and Host Config is able to predict the optimum processor configuration while taking the previously mentioned restriction into account. This is the only SVM in this branch of our predictive model, producing the final System Config output for this branch.

Accelerator Config Another branch of our predictive model starts here. Contrary to the previous branch, Accelerator Config predicts the optimum accelerator configuration only, the optimum host configuration will be predicted by Host Config, which is the next SVM in this branch.

Host Config As the final SVM in this branch, Host Config predicts the best host configuration. The output of this SVM and Accelerator Config is then combined to produce the final System Config output for this branch of the model.

\subsection{Training the Predictor}

Our method for training the predictive models is shown in Figure 5. To train a new predictor we first need to find the best processor configuration for each of our training OPENCL kernels, and extract features. We then use this set of data and classification labels to train our predictor model.

Generating Training Data We use leave-one-out-cross validation for training. This standard machine learning technique works by selecting one benchmark for testing and using the remaining ones for training. To generate the training data for our model we used two benchmark suites: Rodinia [5] and Parboil [36]. In total, we used 32 OPENCL kernels from 15 benchmarks. These benchmarks cover a wide variety of domains including: image processing, data mining, pattern recognition, physics simulations, and linear algebra. We execute each kernel and benchmark a number of times until 
the gap of the upper and lower confidence bounds is smaller than $5 \%$ under a $95 \%$ confidence interval setting. To reduce noise in our performance and energy consumption readings we generate our training data on a unloaded machine, this should not be a problem as we expect model training to be a one-off cost which is carried out at the factory, that is, before a process architecture is released for sale. However, if the hardware configuration changes, i.e. one or more processors are added or removed, new training data will need to generated and the model re-trained. The set of frequencies to be used for generating the training data is decided beforehand. For our hardware platform we chose to use steps of $200 \mathrm{Mhz}$ for each processor configuration, e.g. 200Mhz, 400Mhz, up to 2.0Ghz. We exhaustively execute each OPENCL kernel across all of our considered processor configurations, and record the performance and energy consumption of each. Next, we record the best performing processor configuration for each OPENCL kernel and optimization metric, keeping a label of each. Finally, we extract the values of our selected set of features from each OPENCL kernel; our choice of features is described in more detail in Section 5.3.

Building The Model The processor configuration labels, along with their corresponding feature set, are passed to our supervised learning algorithm. The learning algorithm tries to find a correlation between the feature values and optimal processor configuration labels. The output of our learning algorithm is a version of our SVM based model. Because we target three optimization metrics in this paper, we have constructed three predictive models - one for each of our optimization metrics. In our case, the overall training process (which is dominated by training data generation) takes less than a week on a single machine.

Total Training Time The total training time of our model is comprised of two parts: gathering the training data, and then building the model. Gathering the training data consumes most of the total training time, in this paper it took around 3 days. In comparison actually building the model took a negligible amount of time, less than 1 minute.

\subsection{Features}

Our predictive models are based exclusively on code features of the target OPENCL kernel. The features are extracted using a pass working on the LLVM IR. Since our goal is to develop a portable, architecture-independent approach, we do not use any hardwarespecific features.

We considered a total of 22 candidate raw features (Table 2) in this work. Some features were chosen from our intuition based on factors that can affect kernel mapping e.g. transfer_size and num_float_inst, other features were chosen based on previous work $[13,26]$. Altogether, our candidate features should be able to represent the intrinsic parts of each OPENCL kernel.

Feature Selection In order to build an accurate predictive model through supervised learning the training sample size typically needs to be at least one order of magnitude greater than the number of features. We currently have 32 OPENCL kernels and 22 features, so we would like to reduce the number of features in use. Our process for feature selection is fully automatic, described as follows. Initially, we reduced our feature count by combining several features to form a set of combined normalized features, shown in Table 3 , which are able to carry more information than their parts. Next, we removed any features which carried very similar information as our combined features or their parts, making them redundant. To find which features are closely correlated we constructed a correlation coefficient matrix, which is able to quantify the correlation between any two features. We used Pearson product-moment correlation coefficient. As input, two features are given, a value between +1 and -1 is returned. The closer a coefficient is to $+/-1$, the stronger the
Table 4: Correlations of removed features to the kept features. All correlation values are absolute values.

\begin{tabular}{lll}
\hline Kept Feature & Removed Feature & Correlation \\
\hline & num_BB & 0.99 \\
& num_br & 0.99 \\
num_edges & BB_with_O_phi & 0.98 \\
& num_cond_br & 0.95 \\
& num_uncond_br & 0.94 \\
& avg_phi_args & 0.78 \\
avg_BB_preds & avg_BB_succ & 1.00 \\
num_instr & num_unary_inst & 0.93 \\
num_store & num_int_bin_ops & 0.85 \\
num_comp & num_float_inst & 0.74 \\
\hline
\end{tabular}

Table 5: Features which remained after feature selection.

\begin{tabular}{lll}
\hline mem_by_work & comm-comp_ratio & mem_acc_per_instr \\
comp-mem_ratio & perc_coal_mem_acc & avg_BB_preds \\
transfer-work_ratio & num_edges & transfer_size \\
\hline
\end{tabular}

correlation between the two input features. We removed any feature which had a correlation coefficient (taking the absolute value) greater than 0.75 . Table 4 shows all the features which were removed due to a high correlation value with another feature. For example, we can see that num_BB is highly correlated to num_edges, in this case we chose to remove num_BB from our feature collection. Our feature selection process results in just 9 features remaining for use, these are listed in Table 5. It is to note that our approach for feature selection is automatic. This means the approach can be applied to other sets of candidate features.

Feature Scaling Before the chosen features can be given to our predictive model for training they need to be scaled to a common range. We scaled each of our features between the range of 0 and 1. To scale features extracted from a new kernel during runtime deployment we record the maximum and minimum value of each feature before scaling.

\subsection{Runtime Deployment}

Once we have built and trained our predicted models as described above, we can use them to quickly and efficiently predict the best processor configuration for any new, unseen OPENCL kernel.

We implemented our approach as an OpenCL library extension, building on standard OPENCL APIs. The kernel code will be translated into LLVM IR when the OPENCL API clBuildProgram is invoked by the host code. Once the kernel function is launched through clEnqueueNDRangeKernel, our extension extracts and scales all features needed for the predictive model. Given an optimization goal our extension will choose the correct predictive model to predict the optimal processor configuration. This prediction is then passed to the runtime library to configure the hardware. It is to note that we use the proprietary OPENCL compiler to compile the kernel for the target hardware. The overhead of extracting features, making predictions, and processor configuration is small, which is included in our experimental results.

\section{Experimental Setup \\ 6.1 Platform and Benchmarks}

Hardware Our hardware evaluation platform is an Odroid XU3 big.LITTLE embedded development board. The board has 2 GB LPDDR3 RAM and 64 GB eMMC storage. Table 6 gives detailed information of the hardware platform. We chose this hardware platform as it is representative of the big.LITTLE architecture, provides us with OPENCL implementations for the CPU and GPU, and allows us to extract real-time energy readings. Our reasons for choosing this platform are given in Section 3. 
Table 6: Hardware platform

\begin{tabular}{llll}
\hline & big CPU & LITTLE CPU & GPU \\
\hline Model & Cortex-A15 & Cortex-A7 & Mali-T628 \\
Core Clock & $2.0 \mathrm{GHz}$ & $1.4 \mathrm{GHz}$ & $533 \mathrm{MHz}$ \\
Core Count & 4 & 4 & 6 \\
\hline
\end{tabular}

Systems Software Our platform runs Ubuntu 14.04 Linux with a Heterogeneous Multi-Processing (HMP) scheduler. The scheduler allows us to use the heterogeneous cores at the same time. Our host compiler is gcc v5.4.0, with "-O3" as the compiler option. To use OPENCL on the GPU we use the ARM Mali OPENCL SDK. To use OPENCL on the CPU we use PoCL [18], an OPENCL implementation for CPUs that is based on LLVM. The PoCL compiler automatically applies a set of LLVM-based code transformations to optimize the GPU-tuned OPENCL kernel function for the host CPU.

Benchmarks We used a total of 32 OPENCL kernels from 15 benchmarks. From the Rodinia benchmark suite v2.0.1, we used 22 kernels from 9 benchmarks, and from the Parboil OPENCL benchmark suite, we used 10 kernels from 6 benchmarks. Some benchmarks had to be left out as they were either not compatible with our hardware, or not compatible with our OPENCL compilers.

\subsection{Evaluation Methodology}

Model Evaluation We use leave-one-out cross-validation to evaluate our machine learning model. This means we train our model on 14 benchmarks and apply it to the testing program. We repeat this process 15 times, one for each of the 15 benchmarks. It is a standard evaluation methodology, providing an estimate of the generalization ability of a machine-learning model in predicting unseen data.

Comparisons We compare our approach to another machine learning based approach which provides a portable mapping of OPENCL kernels for heterogeneous systems [13], referred to as PKM hereafter. It is currently the closest work to our own. PKM uses a decision tree to predict whether a given OPENCL program should run on the GPU or the CPU host to achieve the best performance speed-up. We also compare our work to a perfect predictor, referred to as an Oracle hereafter. The Oracle predictor, named after its ability to make prophetic predictions, is able to predict the best possible configuration for all kernels and optimization targets.

Performance Report We profiled each kernel under a processor configuration multiple times and report the geometric mean of each metric. To determine how many runs are needed, we calculated the confidence range using a $95 \%$ confidence interval and make sure that the difference between the upper and lower confidence bounds is smaller than $5 \%$. To eliminate the impact of outliers, we also report harmonic means and median values across kernels and benchmarks. We used the on board energy sensors to measure the entire system. These sensors have been checked against external power measurement instruments and proven to be accurate [17]. To measure the energy consumption, we have developed a lightweight runtime to take readings from the on-board energy sensors at a frequency of 10 samples per second. We then matched the readings against the timestamps of the kernel to calculate the energy consumption.

\section{Experimental Results}

In this section, we compare our work against PKM, showing how our work compares to comparative work. Next, we evaluate our approach against an ideal predictor, an Oracle, showing that our approach can deliver over $93 \%$ of the Oracle's optimizing capability. Finally, we investigate the impact of different input sizes on our model.

\subsection{Overall Performance}

We compare our approach against PKM on runtime (Figure 6), energy consumption (Figure 7), and EDP (Figure 8). The baseline is to offload all kernels on to the big GPU, $C P U_{B, 2.0 G h z}-G P U_{b}$. First, we deeply analyse our results on a per-kernel basis, then we summarize these results on a per-benchmark basis.

Runtime Figure 6 shows the performance achieved by each method when optimizing for runtime, i.e. when a fast response time is the priority. For this metric, the default method of offloading all kernels on to the big GPU already provides near optimum results for most kernels. This is not a surprising result as the OPENCL benchmarks have been heavily tuned for runtime on GPUs. For this metric, PKM is able to select the correct configuration for most of the kernels, but can lead to significant slowdowns for some. For example, it gives 1.6x and 5x slowdown for srad.k1 and hotspot.k1 respectively, by predicting the wrong processor to use. Our approach, by contrast, never gives any slow-down in execution time. In fact, by predicting to use the CPU, our approach is able to deliver over $15 \%$ (up to $80 \%$ ) speed-up for some of the kernels which do not benefit from the GPU execution. Overall, our approach gives an average speed-up of $9.4 \%$.

Energy Consumption Figure 7 compares our approach against PKM when optimizing for energy consumption, i.e. when trying to preserve battery life is the main priority. Both methods are able to reduce energy consumption for more than half of the kernels. For this metric, using the power-hungry GPU is not always desired. For some of the kernels, PKM delivers rather poor energy efficiency performance, consuming up to $8 \mathrm{x}$ more energy, because it maps the kernel to run on a device with a much longer execution time than the big GPU. Comparing to PKM, while our approach also gives the wrong prediction for some of the kernels, e.g. mri-q.k2 and histo.k3, the performance degradation is modest (11\% and $2 \%$ respectively). We believe the prediction accuracy of our model can be improved by using more examples during training. On average, PKM achieves a $28 \%$ improvement for this metric, which is again outperformed by our approach that gives a $45 \%$ improvement. This means that our approach outperforms PKM by a factor of 1.6x when optimizing for energy consumption.

EDP Figure 8 shows the performance achieved by each method when optimizing for EDP, i.e. trying to reduce energy consumption without significantly increasing execution time. Both methods are able to achieve some performance increase over the baseline for EDP. While both approaches are not able to achieve a performance increase every time, our approach limits its performance degradation to $-12 \%$, whereas PKM reaches $-9.7 \mathrm{x}$ for $\mathrm{cfd} . \mathrm{k} 5$ and $-8.1 \mathrm{x}$ for stencil.k1. This huge decrease in EDP can be explained by PKM predicting these benchmarks to be offloaded to the CPU incorrectly, which gives a significantly longer execution time over the baseline. Overall, PKM fails to deliver any improved performance for EDP ($19 \%$ ). Our approach, however, is able to give an average performance increase of $32 \%$ (up to $96 \%$ ), with a significant improvement for the majority of benchmarks. PKM is only able to slightly outperform our approach in one instance for EDP optimization; it is caused by our approach incorrectly predicting the host device.

\subsection{Comparing with the Oracle}

Figures 6 - 8 also compare our approach against the performance achieved by using an ideal predictor (Oracle) for all three evaluation metrics. This comparison shows how close our approach is to the theoretically perfect solution. Our approach is able to either match, or come very close to match, with the Oracle's performance in most cases. Overall, we are able to achieve 93.9\%, 96.8\%, and $96.1 \%$ of the Oracle's optimizing capability for performance, energy consumption, and EDP respectively. We could further improve 


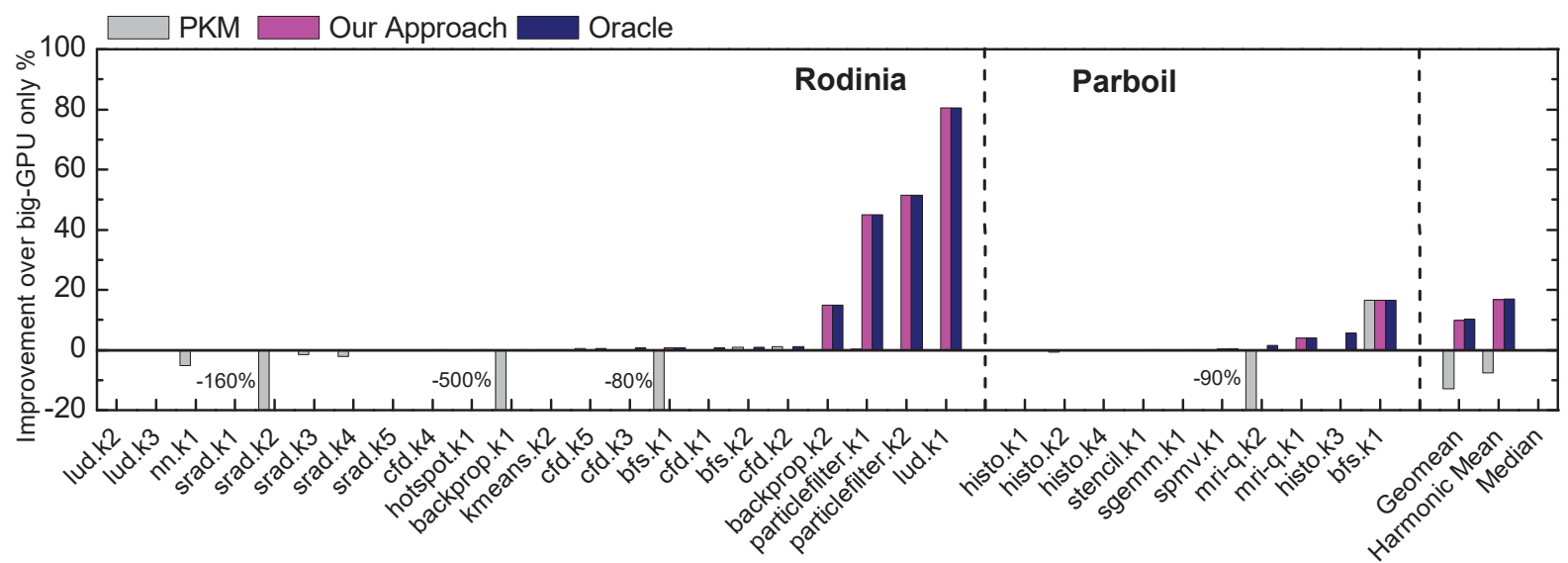

Figure 6: Optimization for runtime for each kernel. Our approach outperforms PKM and does not slowdown any programs. We achieve, on average, $93.9 \%$ of the Oracle performance for this metric.

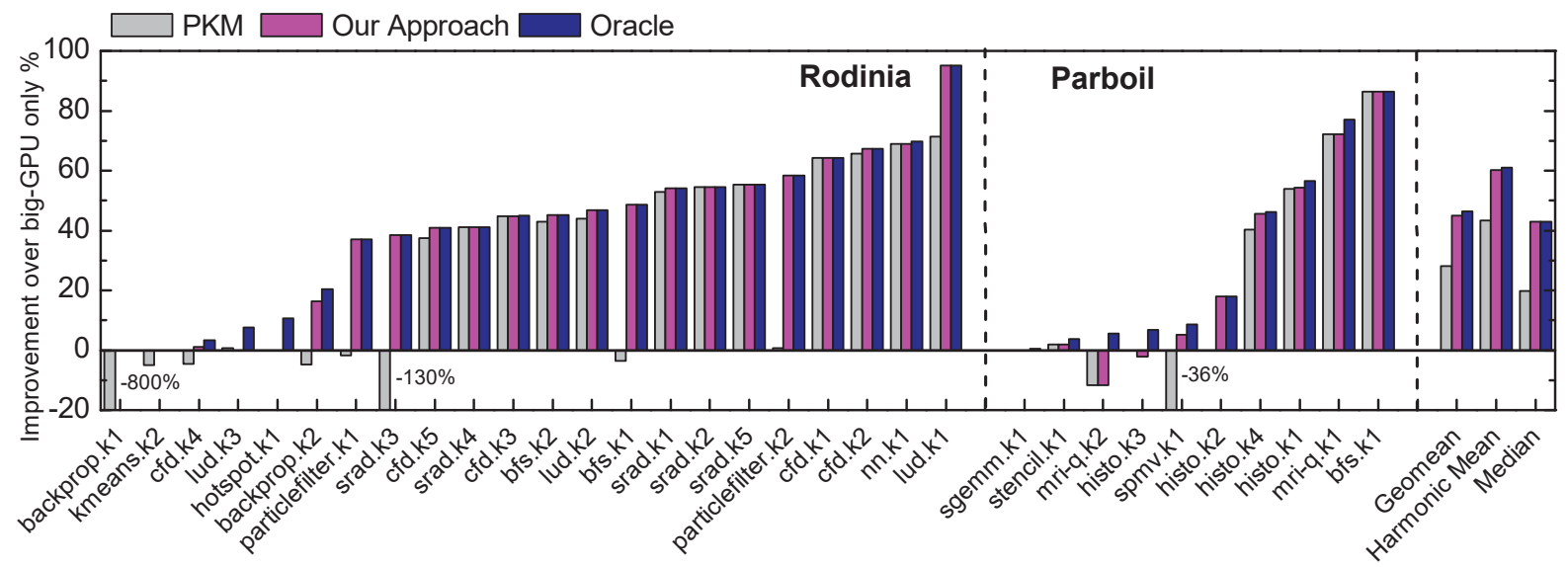

Figure 7: Optimizing for energy consumption for each kernel. Our approach outperforms PKM and only uses more energy in one kernel compared to the baseline. We achieve, on average, $96.8 \%$ of the Oracle performance for this metric.

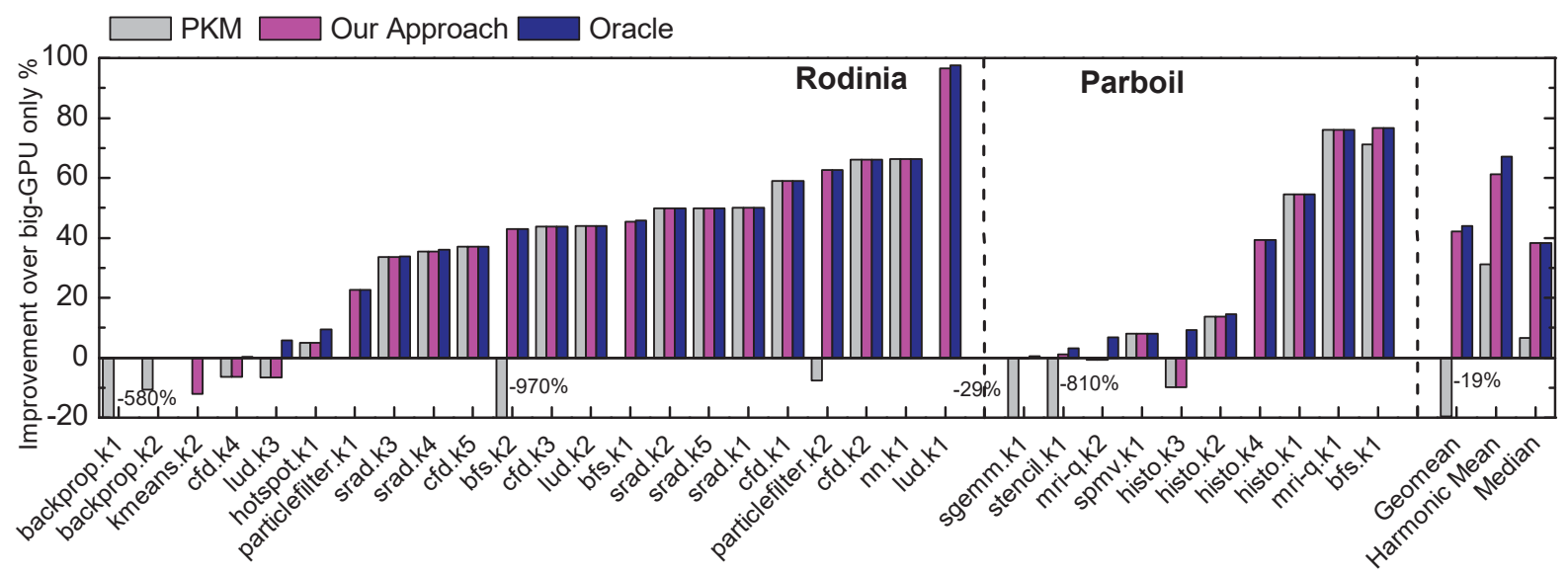

Figure 8: Optimization for EDP for each kernel. Our approach outperforms PKM and only leads to a small increase in EDP when compared to the baseline for a few kernels. We achieve, on average, $96.1 \%$ of the Oracle performance for this metric.

the performance of our predictive model by increasing the accuracy of host processor configuration predictions. We suggest that this would be possible through the introduction of more/different features which are capable of characterizing the host program better than we are currently able. Our model could also be improved through the inclusion of more OPENCL kernels to allow us to better train our models. There could be cases where a model cannot be trained because of a lack of data, this can be solved by including more, and a wider range, of kernels [8].

\subsection{Improvement Per Benchmark}

Figures 9 to 11 compare our performance improvement on a perbenchmark basis against PKM and the Oracle predictor. Similar to comparisons on a per-kernel basis we are able to consistently outperform PKM while achieving close to the Oracle's performance. 


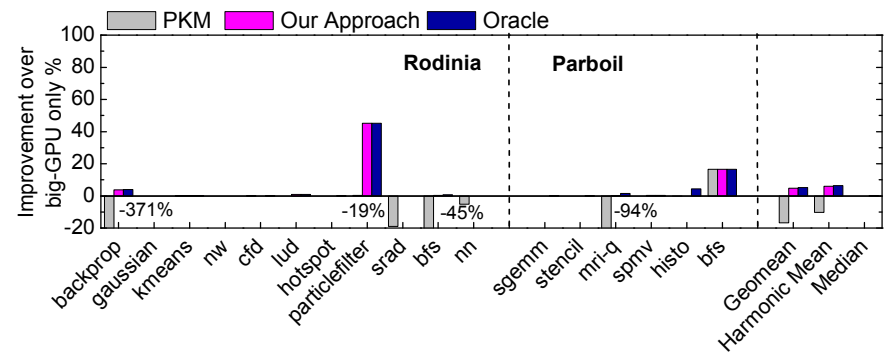

Figure 9: Optimization for runtime for each benchmark. Our approach outperforms PKM and does not slowdown any programs. We achieve, on average, $92.6 \%$ of the Oracle performance for this metric.

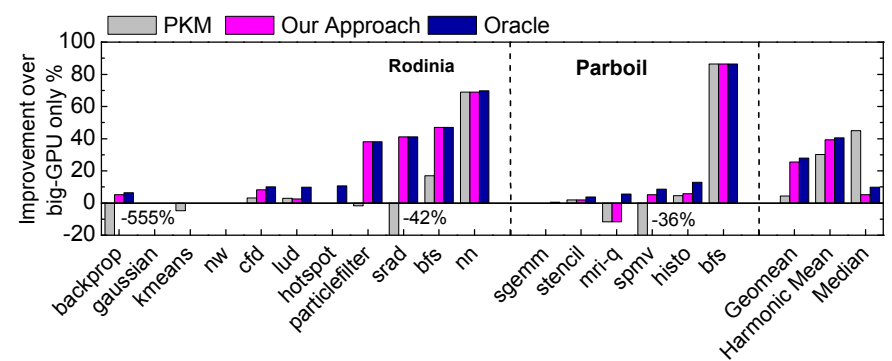

Figure 10: Optimizing for energy consumption for each benchmark. Our approach outperforms PKM and only one benchmark consumes more energy compared to the baseline. We achieve, on average, $91.4 \%$ of the Oracle performance for this metric.

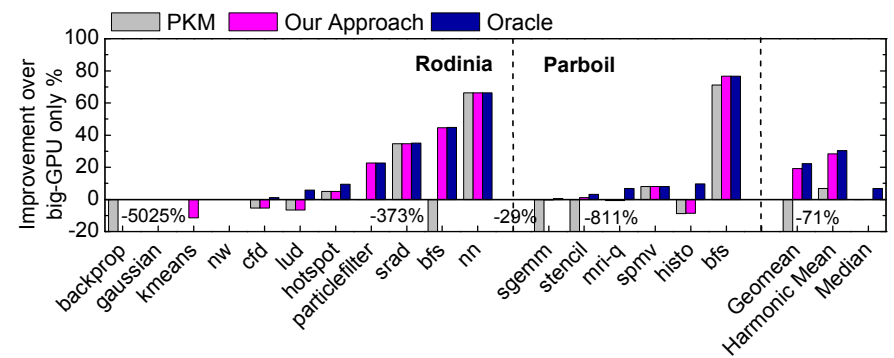

Figure 11: Optimization for EDP for each benchmark. Our approach outperforms PKM and only leads to a small increase in EDP when compared to the baseline for a few benchmarks. We achieve, on average, $84.5 \%$ of the Oracle performance for this metric.

We achieve $92.6 \%, 91.4 \%$, and $84.5 \%$ of the Oracle's optimizing capability for performance, energy consumption, and EDP respectively. Comparing on a per-benchmark basis shows our model's capability to achieve high levels of the Oracle's performance not only for each kernel but for the whole benchmark, i.e. while taking every kernel execution into account.

\subsection{Prediction Accuracy}

Our predictive model is comprised of 4 SVMs organized in a hierarchical structure (Figure 5, Section 5.1). Each SVM is trained separately on the subset of data which is relevant to it, i.e. SVM Accelerator Config is only trained on the kernels which run best on the accelerator, whereas Initial is trained on all kernels. Overall, our predictive model achieves a high accuracy. The predictive models for performance and energy consumption are able to achieve $100 \%$ prediction accuracy. Any reduction in performance when compared to the Oracle are due to assumptions about the Host Device's frequency, that is, some frequencies of the same host are considered as one to help train our model, e.g. kernels with optimum configurations of $C P U_{L, 1.2 G h z}-C P U_{b}$ and $C P U_{L, 1.4 G h z}-$

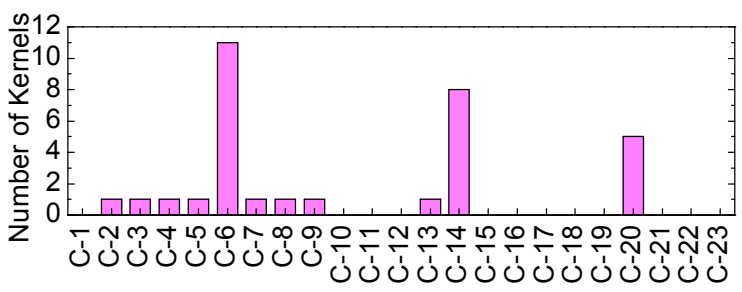

(a) Runtime

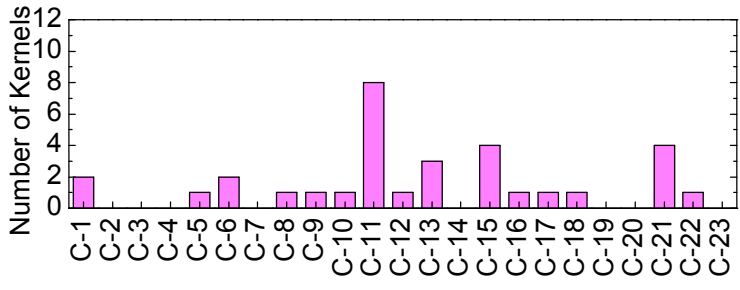

(b) Energy consumption

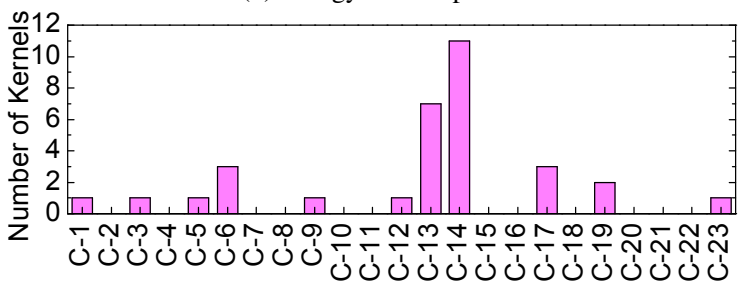

(c) EDP

Figure 12: The number of kernels which benefit from each of the processor configurations listed in Table 7. These histogram diagrams show that the optimal processor configuration depends on the optimization goal.

$C P U_{b}$, are all considered as $C P U_{L, 1.4 G h z}-C P U_{b}$. The predictive model for EDP was able to achieve a correct match for 30 of our 32 kernels, achieving a prediction accuracy of $93.6 \%$.

For EDP Inital, Accelerator and Host Config, and Accelerator Config SVMs all achieve 100\% accuracy. Only the final SVM, Host Config, is unable to achieve $100 \%$ accuracy, although, at this point in our model an incorrect prediction does not yield catastrophic results. Both lud.k3 and histo.k3 achieve their best performance when hosted by the big CPU, but both are predicted to be hosted by the little CPU. We speculate that this difficultly to accurately predict the host comes as a result of our compact set of features (due to the small number of training programs). However, these features are able to represent the intrinsic parts of each kernel, so are able to accurately predict the best device to execute each kernel. Beyond our feature transfer_size, little information is given to the SVM to characterize how the host program of each kernel will perform. Including more features, perhaps just to this final model, which are able to characterize how the host program performs is likely to solve this issue.

\subsection{Optimal Processor Configurations}

Figure 12 shows the number of kernels which perform best on each of the processor configurations we found useful in this work. Table 7 shows how each Config-Num corresponds to a processor configuration, any configuration not included in this table never yielded an optimum result when optimizing any of our kernels for any of our metrics.

It can be observed that there is not a singular configuration which gives an optimum result over many kernels across performance, energy consumption and EDP. For example, C-20 (CPU $U_{B, 2.0 G h z}-$ $\left.G P U_{b}\right)$ is an optimum configuration for 11 of our 32 kernels when optimizing for performance. However, if we used C-20 to optimize 
Table 7: Useful processor configurations in this work.

\begin{tabular}{ll}
\hline Config-Num & Configuration \\
\hline$C-1$ & $C P U_{B, 400 M h z}-G P U_{b}$ \\
$C-2$ & $C P U_{B, 1.2 G h z}-G P U_{b}$ \\
$C-3$ & $C P U_{B, 1.4 G h z}-G P U_{b}$ \\
$C-4$ & $C P U_{B, 1.6 G h z}-G P U_{b}$ \\
$C-5$ & $C P U_{B, 1.8 G h z}-G P U_{b}$ \\
$C-6$ & $C P U_{B, 2.0 G h z}-G P U_{b}$ \\
$C-7$ & $C P U_{B, 1.8 G h z}-G P U_{l}$ \\
$C-8$ & $C P U_{B}, 2.0 G h z-G P U_{l}$ \\
$C-9$ & $C P U_{L}, 400 M h z-G P U_{b}$ \\
$C-10$ & $C P U_{L}, 600 M h z-G P U_{b}$ \\
$C-11$ & $C P U_{L}, 800 M h z-G P U_{b}$ \\
$C-12$ & $C P U_{L}, 1.0 G h z-G P U_{b}$ \\
$C-13$ & $C P U_{L, 1.2 G h z}-G P U_{b}$ \\
$C-14$ & $C P U_{L}, 1.4 G h z-G P U_{b}$ \\
$C-15$ & $C P U_{L, 800 M h z}-G P U_{l}$ \\
$C-16$ & $C P U_{L}, 1.2 G h z-G P U_{l}$ \\
$C-17$ & $C P U_{L, 1.4 G h z}-G P U_{l}$ \\
$C-18$ & $C P U_{B, 1.0 G h z}-C P U_{b}$ \\
$C-19$ & $C P U_{B, 1.6 G h z}-C P U_{b}$ \\
$C-20$ & $C P U_{B, 2.0 G h z}-C P U_{b}$ \\
$C-21$ & $C P U_{L, 800 M h z}-C P U_{l}$ \\
$C-22$ & $C P U_{L, 1.0 G h z}-C P U_{l}$ \\
$C-23$ & $C P U_{L, 1.2 G h z}-C P U_{l}$ \\
\hline
\end{tabular}

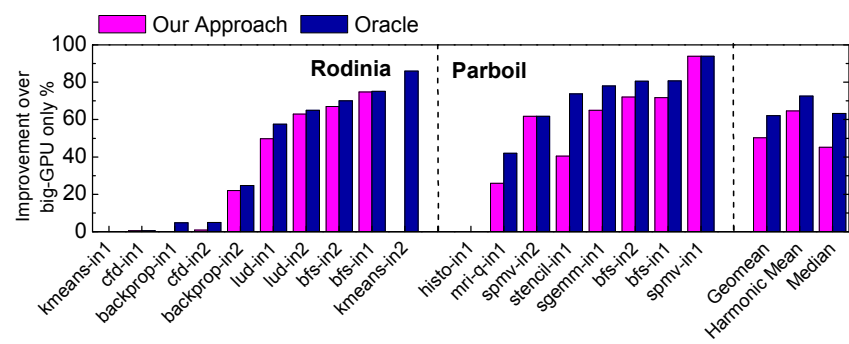

Figure 13: Optimization for EDP. Performance of our model compared to the Oracle when different inputs are considered. Benchmarks names have either -in1 or -in2 appended to denote the different input sizes.

for energy consumption or EDP we would only achieve an optimum result for 4 and 3 kernels, respectively. Each configuration which performs well for one optimization goal does not perform well for the others. We can also see that the best number of kernels a configuration can optimize for at once is $11(34 \%)$, and that there is a wide range of configurations which give optimizing performance for each metric. This shows the need for an approach that is able to spot the subtle differences in each kernel to optimize correctly.

\subsection{Varying Input Sizes}

Figure 13 shows how our model performs when we consider different inputs. Only some of the benchmarks provided us with methods to change their input. We have presented these results on a per-benchmark basis for EDP. However, the results per-kernel, and for each metric are very similar.

It can be observed that our approach performs well, even when optimizing the same benchmark with different inputs. In fact, for EDP, our model never gives a slowdown. Overall, we achieve $90 \%$ of the Oracle performance. Our approach is successful when input sizes differ as a benchmark's input size will alter the execution of a kernel, thus, changing some features. Our model will treat this as a new, unseen kernel.

\section{Model Analysis}

In this section we analyse the overall effectiveness of our model. First, we analyse the importance of each of our chosen features for each of our evaluation metrics. We then compare our model against other widely used machine learning classification techniques.

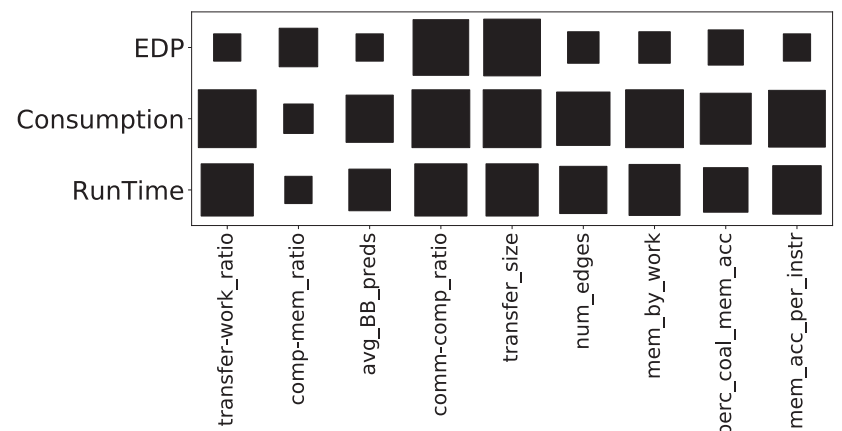

Figure 14: A Hinton diagram showing how each selected feature is likely to impact the performance for each model. Here, the larger the box, the more important a feature is.

Finally, we analyse the training and deployment overhead of using our approach.

\subsection{Feature Importance}

Figure 14 shows a Hinton diagram illustrating the importance of our features. The impact each feature has on each of our predictive models, for performance, energy consumption and EDP, can easily be seen. Here, the larger the box, the more significant a particular feature's contribution to the prediction accuracy is. Along the $\mathrm{x}$-axis is each feature, and along the y-axis is each metric, corresponding to each of our models. We calculate the importance of each metric though the information gain ratio.

It can be observed that comm-comp_ratio and transfer_size are important features when determining the correct processor configuration, independent of the optimizing metric. Each feature has a different level of importance for each metric, e.g. transferwork_ratio is extremely important when optimizing for Energy Consumption, less important for runtime, and has little importance for EDP. This diagram shows the need for distinct models for different optimization goals.

\subsection{Alternative Predictive Modeling Techniques}

Figure 15 shows the geometric mean of improvement of each kernel from the baseline achieved by our approach and four widely used classification techniques: Multi-layer Perceptron (MLP), K-Nearest Neighbours (KNN), Logistic Regression, and Naive Bayes. Each of the alternate predictive modeling techniques were trained and evaluated using the same methods and training data as our model.

Our approach outperforms each alternate technique for every optimization metric. None of the alternate techniques were able to achieve a positive geometric mean when optimizing for runtime, and were unable to yield better optimization results for any of the kernels or metrics we considered. This figure shows the success of our approach against alternate techniques. It is to note that the performance of these alternate modeling techniques may improve if there are more training examples to support the use of a richer set of features. However, we found that our hierarchical SVM based approach performs well on the available benchmarks.

\subsection{Training and Deployment Overhead}

Our models are trained offline with training examples. In this work, collecting the examples took three days using one platform, which has no impact on runtime cost. The overhead of using the trained models includes extracting program features and making predictions. These processes take place during the just-in-time compilation of the OPENCL kernels. This overhead is negligible, approximate $10 \mathrm{~ms}$ in total. 


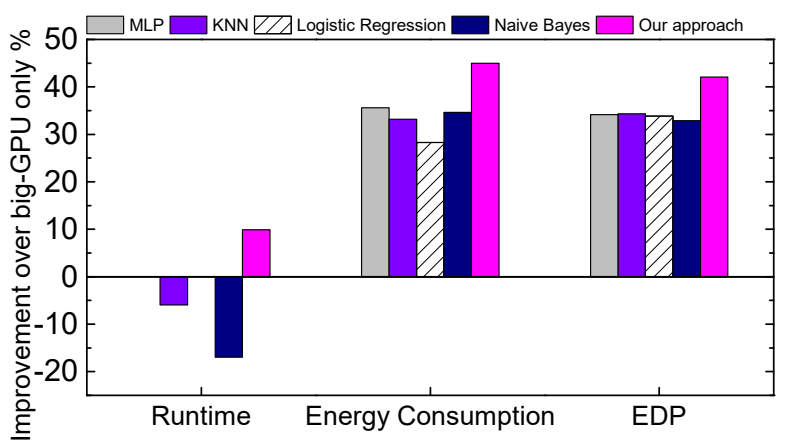

Figure 15: Comparisons to other predictive modeling techniques. Our hierarchical SVM based approach delivers the best overall performance.

\section{Conclusion}

This paper has presented an automatic approach to map OPENCL tasks on heterogeneous mobile platforms, providing a significant performance improvement over comparative works. Central to our approach is a unified, machine learning based framework that predicts, for a given optimization criterion, which of the processors of the system to use to run the OPENCL program, and the clock frequency of the processor. The prediction is based on a set of code and runtime features of the program. Our model is built and trained off-line, and is fully automatic. We evaluate our approach on an ARM big.LITTLE mobile platform using a set of OPENCL benchmarks from the Rodina and the Parboil benchmark suites. Experimental results show that our approach consistently outperforms a comparative OPENCL mapping technique across three evaluation metrics: runtime, energy consumption and EDP. This translates to, on average, above $93 \%$ of the performance given by an ideal predictor.

\section{Acknowledgement}

The research was partly supported by the UK Engineering and Physical Sciences Research Council (EPSRC) under grant agreements EP/M01567X/1 (SANDeRs) and EP/M015793/1 (DIVIDEND).

\section{References}

[1] H. Almatary et al. Reducing the implementation overheads of ipcp and dfp. In RTSS '15.

[2] J. Ceng et al. Maps: an integrated framework for mpsoc application parallelization. In $D A C$ ' 08 .

[3] P. Chakraborty et al. Opportunity for compute partitioning in pursuit of energy-efficient systems. In LCTES 2016.

[4] K. Chandramohan and M. F. O'Boyle. Partitioning data-parallel programs for heterogeneous mpsocs: time and energy design space exploration. In LCTES 2014.

[5] S. Che et al. Rodinia: A benchmark suite for heterogeneous computing. In IISWC '09.

[6] G. Chen et al. Effisha: A software framework for enabling effficient preemptive scheduling of gpu. In $P P O P P^{\prime} 17$.

[7] Y. Cho et al. Energy-reduction offloading technique for streaming media servers. Mobile Information Systems, 2016.

[8] C. Cummins et al. Synthesizing benchmarks for predictive modeling. In CGO 2017.

[9] K. Dev and S. Reda. Scheduling challenges and opportunities in integrated cpu+gpu processors. In ESTIMedia'16.

[10] M. K. Emani et al. Smart, adaptive mapping of parallelism in the presence of external workload. In $C G O, 2013$.

[11] S. Eyerman and L. Eeckhout. Probabilistic job symbiosis modeling for smt processor scheduling. In ASPLOS XV, 2010
[12] E. Garzón et al. An approach to optimise the energy efficiency of iterative computation on integrated gpu-cpu systems. The Journal of Supercomputing, 2016.

[13] D. Grewe et al. Portable mapping of data parallel programs to opencl for heterogeneous systems. In $C G O$ ' 13 .

[14] D. Grewe et al. A workload-aware mapping approach for data-parallel programs. In HiPCA, 2011.

[15] D. Grewe et al. Opencl task partitioning in the presence of gpu contention. In $L C P C, 2013$.

[16] N. Guan et al. Schedulability analysis of preemptive and nonpreemptive edf on partial runtime-reconfigurable fpgas. ACM TODAES, 2008

[17] C. Imes and H. Hoffmann. Bard: A unified framework for managing soft timing and power constraints. In SAMOS, 2016.

[18] Jääskeläinen et al. Pocl: A performance-portable opencl implementation. Int. J. Parallel Program., 2015.

[19] W. Jia et al. Gpu performance and power tuning using regression trees. ACM Trans. Archit. Code Optim., 2015.

[20] R. Kaleem et al. Adaptive heterogeneous scheduling for integrated gpus. In $P A C T$ ' 14

[21] S. S. Latifi Oskouei et al. Cnndroid: Gpu-accelerated execution of trained deep convolutional neural networks on android. In $M M$ ' 16 2016.

[22] J. Lee, M. Samadi, and S. Mahlke. Orchestrating multiple data-parallel kernels on multiple devices. In PACT'15,

[23] M. S. Lee et al. Accelerating bootstrapping in fhew using gpus. In ASAP'15,

[24] J. Leng et al. Gpuwattch: enabling energy optimizations in gpgpus. In ISCA 2013.

[25] K. Ma et al. Greengpu: A holistic approach to energy efficiency in gpu-cpu heterogeneous architectures. In ICPP 2014

[26] A. Magni et al. Automatic optimization of thread-coarsening for graphics processors. In $P A C T$ ' 14 .

[27] D. Majeti et al. Automatic data layout generation and kernel mapping for cpu+gpu architectures. In CC 2016.

[28] P.-J. Micolet et al. A machine learning approach to mapping streaming workloads to dynamic multicore processors. In LCTES 2016.

[29] W. Ogilvie et al. Minimizing the cost of iterative compilation with active learning. In $C G O, 2017$.

[30] P. Pandit and R. Govindarajan. Fluidic kernels: Cooperative execution of opencl programs on multiple heterogeneous devices. In CGO ' 14 .

[31] H. Park et al. Zero and data reuse-aware fast convolution for deep neural networks on gpu. In CODES+ISSS 2016.

[32] J. Ren et al. Optimise web browsing on heterogeneous mobile platforms: a machine learning based approach.

[33] J. Ren et al. Optimise web browsing on heterogeneous mobile platforms: a machine learning based approach. In INFOCOM, 2017.

[34] A. K. Singh, M. Shafique, A. Kumar, and J. Henkel. Mapping on multi/many-core systems: Survey of current and emerging trends. In DAC 2013.

[35] A. Snavely and D. M. Tullsen. Symbiotic jobscheduling for a simultaneous multithreaded processor. In ASPLOS IX, 2000.

[36] J. A. Stratton and others. Parboil: A revised benchmark suite for scientfic and commercial throughput computing.

[37] G. Tournavitis et al. Towards a holistic approach to auto-parallelization: integrating profile-driven parallelism detection and machine-learning based mapping. In PLDI '09.

[38] S. Verdoolaege et al. Polyhedral parallel code generation for cuda. ACM TACO, 2013

[39] Z. Wang and M. O'Boyle. Mapping parallelism to multi-cores: a machine learning based approach. In $P P O P P^{\prime} 09$.

[40] Z. Wang and M. F. O'Boyle. Partitioning streaming parallelism for multi-cores: a machine learning based approach. In PACT, 2010.

[41] Z. Wang et al. Integrating profile-driven parallelism detection and machine-learning-based mapping. ACM TACO, 2014.

[42] Z. Wang et al. Automatic and portable mapping of data parallel programs to opencl for gpu-based heterogeneous systems. ACM TACO 2015.

[43] Y. Wen et al. Smart multi-task scheduling for opencl programs on cpu/gpu heterogeneous platforms. In HiPC, 2014. 